Journal of Engineering and Applied Sciences 14 (Special Issue 9): 10569-10571, 2019

ISSN: 1816-949X

(C) Medwell Journals, 2019

\title{
Convergence Weibull Distribution to Normal Distribution by using Differential Geometry
}

\author{
Wasan Abbas Jasim \\ Department of Applied Geology, College of Science, University of Babylon, Babil, Iraq
}

\begin{abstract}
In this research, we use the differential geometry to show that Weibull distribution converges to normal distribution by connecting between differential geometry and statistics. We apply the following equation:

$$
\mathrm{k}=\frac{1}{\mathrm{D}}\left[\frac{\partial}{\partial \mathrm{v}}\left(\frac{\mathrm{D}}{\mathrm{E}} \Gamma_{11}^{2}\right)-\frac{\partial}{\partial \mathrm{u}}\left(\frac{\mathrm{D}}{\mathrm{E}} \Gamma_{12}^{2}\right)\right]=\frac{1}{\mathrm{D}}\left[\frac{\partial}{\partial \mathrm{u}}\left(\frac{\mathrm{D}}{\mathrm{G}} \Gamma_{12}^{1}\right)-\frac{\partial}{\partial \mathrm{v}}\left(\frac{\mathrm{D}}{\mathrm{G}} \Gamma_{12}^{1}\right)\right]
$$

to calculate the Gaussian curvature $(\mathrm{k})$ for Weibull distribution in the case of parametric lines are not orthogonal and show that if it is convergent to normal distribution by comparing the value of Gaussian curvature for Weibull distribution with the value of Gaussian curvature for the normal distribution.
\end{abstract}

Key words: Calculate, Gaussian, weibull, geometry, curvature, comparing

\section{INTRODUCTION}

This research present some interesting connections between statistics and differential geometry. Also, it contains the result of computing the Gaussian curvature of Weibull distribution in the case of parametric lines are not orthogonal. If the Gaussian curvature of Weibull distribution approaches to the Gaussian curvature of the normal distribution, we say that the distribution converges to normal distribution.

There are some researchers who worked in this field in end of twenty century and beginning of twenty one century. Kass and Vos (1997) used the following equation:

$$
-\frac{1}{\sqrt{\mathrm{EG}}}\left(\frac{\partial}{\partial \mathrm{u}}\left(\frac{1}{\mathrm{E}} \frac{\partial \sqrt{\mathrm{G}}}{\partial \mathrm{u}}\right)+\frac{\partial}{\partial \mathrm{v}}\left(\frac{1}{\sqrt{\mathrm{G}}} \frac{\partial \sqrt{\mathrm{E}}}{\partial \mathrm{v}}\right)\right)
$$

to compute the Gaussian curvature $(\mathrm{K})$ of trinomial and $\mathrm{t}$ families. Chen (2014) used the equation:

$$
\begin{gathered}
\frac{\mathrm{R}_{1212}}{\mathrm{EG}-\mathrm{F}^{2}}=\frac{(12,12)}{\mathrm{EG}-\mathrm{F}^{2}} \text { where }(12,12)=\mathrm{R}_{1212}=\sum_{\mathrm{m}=1}^{2} \mathrm{R}_{121}^{\mathrm{m}} \mathrm{g}_{\mathrm{m} 2} \\
\mathrm{R}_{\mathrm{ijk}}^{1}=\frac{\partial}{\partial \mathrm{u}_{\mathrm{j}}} \Gamma_{\mathrm{ik}}^{\mathrm{i}}-\frac{\partial}{\partial \mathrm{u}_{\mathrm{i}}} \Gamma_{\mathrm{jk}}^{1}+\Gamma_{\mathrm{ik}}^{\mathrm{m}} \Gamma_{\mathrm{mj}}^{1}-\Gamma_{\mathrm{jk}}^{\mathrm{m}} \Gamma_{\mathrm{mi}}^{1}, \text { sum on } \mathrm{m}
\end{gathered}
$$

to compute the Gaussian curvature $(\mathrm{K})$ for the distributions (normal, Cauchy and t family). He showed that in normal distribution Gaussian curvature $\mathrm{K}=-1 / 2$ and in Cauchy distribution $\mathrm{K}=-2$ while in $\mathrm{t}$ family distribution with $\mathrm{r}$ degrees of freedom, he got $\mathrm{K}=-\frac{\mathrm{r}+3}{2 \mathrm{r}}$. In Gruber used the following equation:

$$
\begin{aligned}
& -\frac{1}{2 \sqrt{E G-F^{2}}}\left[\frac{\partial}{\partial u} \frac{G_{u}-F_{v}}{\sqrt{E G-F^{2}}}-\frac{\partial}{\partial v} \frac{F_{u}-E_{v}}{\sqrt{E G-F^{2}}}\right]- \\
& \frac{1}{4\left(\sqrt{E G-F^{2}}\right)^{2}}\left|\begin{array}{ccc}
E & F & G \\
E_{u} & F_{v} & G_{u} \\
E_{v} & F_{v} & G
\end{array}\right|
\end{aligned}
$$

to compute the Gaussian curvature o f gamma family of distributions in the case of parametric lines are orthogonal $(\mathrm{F}=0)$ and showed that Gamma distribution converges to the normal distribution.

Metric Tensor for Weibull distribution (Barndorff-Nielsen et al., 1986; Kass and Vos, 1997; O’ Neill, 1966; Wikipedia, 2005): A random variable $x$ has a Weibull distribution if its probability density function of form:

$$
f(x, u, v)=\frac{u x^{u-1} u^{-\left(\frac{x}{v}\right)^{u}}}{v^{u}}, x>0
$$

Where:

$\mathrm{v}$ : Scale parameter

u : Shape parameter

$$
\mathrm{E}=-\mathrm{E}\left(\frac{\partial^{2} \operatorname{Inf}}{\partial \mathrm{u}^{2}}\right)=\frac{1}{\mathrm{u}^{2}}+\frac{\Gamma_{(2)}^{(2)}}{\mathrm{u}^{2}}=\frac{\Gamma_{(2)}^{(2)+1}}{\mathrm{u}^{2}}=\frac{\mathrm{c}_{1}}{\mathrm{u}_{2}}
$$




$$
\begin{aligned}
& \mathrm{F}=-\mathrm{E}\left(\frac{\partial^{2} \operatorname{Inf}}{\partial \mathrm{v} \partial \mathrm{u}}\right)=\frac{1}{\mathrm{v}}-\frac{1}{\partial} \mathrm{E}\left(\frac{\mathrm{x}}{\mathrm{v}}\right)^{\mathrm{u}}- \\
& \frac{\mathrm{u}}{\mathrm{v}} \mathrm{E}\left[\left(\frac{\mathrm{x}}{\mathrm{v}}\right)^{\mathrm{u}} \operatorname{In} \frac{\mathrm{x}}{\mathrm{v}}\right]=-\frac{\Gamma_{(2)}^{(1)}}{\mathrm{v}}=\frac{\mathrm{C}_{2}}{\mathrm{v}} \\
& \mathrm{G}=-\mathrm{E}\left(\frac{\partial^{2} \operatorname{Inf}}{\partial \mathrm{v}^{2}}\right)=\left(\frac{\mathrm{u}}{\mathrm{v}}\right)^{2} \\
& \psi(2)=\psi(1)+1=-0.5772156649+1=0.422784335=\Gamma_{(2)}^{\prime} \\
& \Gamma_{(2)}^{(2)}=\psi{ }^{\prime}(2)+\left(\Gamma_{(2)}^{\prime}\right)^{2}=0.644934067+ \\
& (0.422784335)^{2}=0.823680661 \\
& \mathrm{c}_{1}=\Gamma_{(2)}^{(2)}+1=1.823680661 \\
& \mathrm{c}_{2}=-\Gamma_{(2)}^{\prime}=-0.422784335 \\
& \mathrm{C}_{3}=\mathrm{c}_{1}-\mathrm{c}_{2}^{2}=1.644934067
\end{aligned}
$$

$$
\text { Metric tensor }=\left[\begin{array}{ll}
\frac{\mathrm{c}_{1}}{\mathrm{u}_{2}} & \frac{\mathrm{c}_{2}}{\mathrm{v}} \\
\frac{\mathrm{c}_{2}}{\mathrm{v}} & \left(\frac{\mathrm{u}}{\mathrm{v}}\right)^{2}
\end{array}\right]
$$

or

$$
\mathrm{ds}^{2}=\frac{\mathrm{c}_{1}}{\mathrm{u}^{2}}(\mathrm{du})^{2}+2 \frac{\mathrm{c}_{2}}{\mathrm{v}} \mathrm{dudv}+\left(\frac{\mathrm{u}}{\mathrm{v}}\right)^{2}(\mathrm{dv})^{2}
$$

The Gaussian curvature of the probability distribution (Do Carmo, 1976): First, we define the six well known Christoffel symbols as:

$$
\begin{aligned}
& \Gamma_{11}^{1}=\frac{\mathrm{GE}_{\mathrm{u}}-2 \mathrm{FF}_{\mathrm{u}}+\mathrm{FV}_{\mathrm{v}}}{2\left(\mathrm{EG}-\mathrm{F}^{2}\right)}, \quad \Gamma_{12}^{2}=\frac{\mathrm{EG}_{\mathrm{u}}-\mathrm{FE}_{\mathrm{v}}}{2\left(\mathrm{EG}-\mathrm{F}^{2}\right)} \\
& \Gamma_{11}^{2}=\frac{2 \mathrm{EF}_{\mathrm{u}}-\mathrm{EE}_{\mathrm{v}}-\mathrm{FE}_{\mathrm{u}}}{2\left(\mathrm{EG}-\mathrm{F}^{2}\right)}, \quad \Gamma_{22}^{1}=\frac{2 \mathrm{GF}_{\mathrm{v}}-\mathrm{GG}_{\mathrm{u}}-\mathrm{FG}_{\mathrm{v}}}{2\left(\mathrm{EG}-\mathrm{F}^{2}\right)} \\
& \Gamma_{12}^{1}=\frac{\mathrm{GE}_{\mathrm{v}}-\mathrm{FG}_{\mathrm{u}}}{2\left(\mathrm{EG}-\mathrm{F}^{2}\right)}, \quad \Gamma_{22}^{2}=\frac{\mathrm{EG}_{\mathrm{v}}-2 \mathrm{FF}_{\mathrm{v}}+\mathrm{FG}_{\mathrm{u}}}{2\left(E G-F^{2}\right)}
\end{aligned}
$$

Since, E, F and $\mathrm{G}$ are functions of parameters (u, v). Now, we select four formulas that can be used to compute the Gaussian curvature of the distributions:

$$
\begin{aligned}
& K=\frac{1}{\sqrt{E G}}\left(\frac{\partial}{\partial u}\left(\frac{1}{\sqrt{E}} \frac{\partial \sqrt{G}}{\partial u}\right)+\frac{\partial}{\partial v}\left(\frac{1}{\sqrt{G}} \frac{\partial \sqrt{E}}{\partial v}\right)\right) \\
& K=-\frac{1}{2 \sqrt{E G-F^{2}}}\left[\frac{\partial}{\partial u} \frac{G_{u}-F_{v}}{\sqrt{E G-F^{2}}}-\frac{\partial}{\partial v} \frac{F_{u}-E_{v}}{\sqrt{E G-F^{2}}}\right]- \\
& \frac{1}{4\left(E G-F^{2}\right)^{2}}\left|\begin{array}{ccc}
E & F & G \\
E_{u} & F_{u} & G_{u} \\
E_{v} & F_{v} & G_{v}
\end{array}\right|
\end{aligned}
$$

$$
\mathrm{K}=\frac{1}{\mathrm{D}}\left[\frac{\partial}{\partial \mathrm{v}}\left(\frac{\mathrm{D}}{\mathrm{E}} \Gamma_{11}^{2}\right)-\frac{\partial}{\partial \mathrm{u}}\left(\frac{\mathrm{D}}{\mathrm{E}} \Gamma_{12}^{2}\right)\right]=\frac{1}{\mathrm{D}}\left[\frac{\partial}{\partial \mathrm{u}}\left(\frac{\mathrm{D}}{\mathrm{G}} \Gamma_{22}^{1}\right)-\frac{\partial}{\partial \mathrm{v}}\left(\frac{\mathrm{D}}{\mathrm{G}} \Gamma_{12}^{1}\right)\right]
$$

where, $\mathrm{D}^{2}=\mathrm{EG}-\mathrm{F}^{2}$

$$
\mathrm{K}=\frac{\mathrm{R}_{1212}}{\mathrm{EG}-\mathrm{F}^{2}}=\frac{(12,12)}{\mathrm{EG}-\mathrm{F}^{2}}
$$

Where $(12,12)=R_{1212}=\sum_{m=1}^{2} R_{121}^{m} g_{m 2}$

$$
\mathrm{R}_{\mathrm{ijk}}^{1}=\frac{\partial}{\partial \mathrm{u}_{\mathrm{j}}} \Gamma_{\mathrm{jk}}^{1}-\frac{\partial}{\partial \mathrm{u}_{\mathrm{i}}} \Gamma_{\mathrm{jk}}^{1}+\Gamma_{\mathrm{mj}}^{1}-\Gamma_{\mathrm{jk}}^{\mathrm{m}} \Gamma_{\mathrm{mi}}^{1} \text {, sumon } \mathrm{m}
$$

where, the quantities of $\mathrm{R}_{\mathrm{ijk} 1}$ are components of a tensor of the fourth order. Notice that $g_{11}, g_{12}$ and $g_{22}$ are simply tensor notation for E, F and G.

Remark: We can not use the equation (A) to compute the Gaussian curvature of Weibull distribution because we take the case that the parametric lines us $\mathrm{v}$ of this distribution are not orthogonal $(\mathrm{F} \neq 0)$.

The gaussian Curvature for Weibull distribution: We use the equation (C) to compute the Gaussian curvature of this distribution. We can find that:

$$
\begin{aligned}
& E_{u}=\frac{2 c_{1}}{u^{3}}, E_{v}=0, F_{u}=0, F_{v}=\frac{c_{2}}{v_{2}}, G_{u}=\frac{2 u}{v^{3}}, G_{v}=\frac{2 u^{2}}{v^{2}} \\
& \Gamma_{11}^{1}=\frac{G E_{u}-2 F F_{u}+F E_{v}}{2\left(E G-F^{2}\right)} \\
& =\frac{\frac{u 2}{v 2}, \frac{-2 c_{1}}{u^{3}}}{2\left[\frac{c_{1}}{u^{2}},\left(\frac{u}{v}\right) 2-\left(\frac{c_{2}}{v}\right) 2\right]}=\frac{\frac{-2 c_{1}}{v^{2} u}}{2\left(\frac{c_{1}}{v^{2}}-\frac{c_{2}^{2}}{v^{2}}\right)}=\frac{\frac{-2 c_{1}}{v^{2} u}}{2\left(\frac{c_{1}-c_{2}^{2}}{v^{2}}\right)}= \\
& -\frac{2 c_{1}}{v^{2} u} \cdot \frac{v^{2}}{2\left(c_{1}-c_{2}^{2}\right)}=-\frac{c_{1}}{c_{g} u}
\end{aligned}
$$

$$
\begin{gathered}
\Gamma_{11}^{2}=\frac{2 \mathrm{EF}_{\mathrm{u}}-\mathrm{EE}_{\mathrm{v}}-\mathrm{FE}_{\mathrm{u}}}{2\left(\mathrm{EG}-\mathrm{F}^{2}\right)}= \\
\frac{-\frac{\mathrm{c}_{2}}{\mathrm{v}}, \frac{-2 \mathrm{c}_{1}}{\mathrm{u}^{3}}}{2\left(\frac{\mathrm{c}_{1}-\mathrm{C}_{2}^{2}}{\mathrm{v}^{2}}\right)}=\frac{2 \mathrm{c}_{1} \mathrm{C}_{2}}{\mathrm{vu}^{9}} \cdot \frac{\mathrm{v}^{2}}{2\left(\mathrm{c}_{1}-\mathrm{C}_{2}^{2}\right)}=\frac{\mathrm{C}_{1} \mathrm{C}_{2} \mathrm{v}}{\mathrm{C}_{9} \mathrm{u}_{9}} \\
\Gamma_{12}^{1}=\frac{G E_{\mathrm{v}}-\mathrm{FG}_{\mathrm{u}}}{2\left(\mathrm{EG}-\mathrm{F}^{2}\right)}=\frac{-\frac{\mathrm{c}_{2}}{\mathrm{v}}, \frac{2 \mathrm{u}}{\mathrm{v}_{2}}}{\frac{2\left(\mathrm{c}_{1}-\mathrm{C}_{2}^{2}\right)}{\mathrm{v}^{2}}}=\frac{-2 \mathrm{C}_{2} \mathrm{u}}{\mathrm{v}^{3}} \cdot \frac{\mathrm{v}^{2}}{2\left(\mathrm{c}_{1}-\mathrm{C}_{2}^{2}\right)}=\frac{\mathrm{C}_{2} \mathrm{u}}{\mathrm{C}_{3} \mathrm{v}}
\end{gathered}
$$




$$
\begin{aligned}
& \Gamma_{12}^{2}=\frac{E G_{u}-F E_{v}}{2\left(E G-F^{2}\right)}=\frac{\frac{c_{1}}{u^{2}}, \frac{2 u}{v^{2}}}{\frac{2\left(c_{1}-c_{2}^{2}\right)}{v^{2}}}=\frac{2 c_{1} u}{u^{2} v^{2}} \cdot \frac{v^{2}}{2 c_{3}}=\frac{c_{1}}{c_{3} u} \\
& \Gamma_{22}^{1}=\frac{2 \mathrm{GF}_{\mathrm{v}}-\mathrm{GG}_{\mathrm{u}}-\mathrm{FG}_{\mathrm{v}}}{2\left(\mathrm{EG}-\mathrm{F}^{2}\right)}= \\
& \frac{\frac{2 u^{2}}{v^{2}}, \frac{c 2}{v^{2}}-\frac{u^{2}}{v^{2}}, \frac{2 u}{v^{2}}-\frac{c_{2}}{v},-\frac{-2 u^{2}}{v^{2}}}{\frac{2 c_{3}}{v^{2}}}=\frac{\frac{-2 u^{2} c_{2}}{v^{4}}-\frac{2 u^{3}}{v^{4}}+\frac{2 c_{2} u^{2}}{v^{4}}}{\frac{2 c_{3}}{v^{2}}}= \\
& \frac{\frac{-2 u^{2} c_{2}-2 u^{3}+2 c_{2} u^{2}}{\frac{v^{4}}{v^{2}}}}{\frac{2 c_{3}}{\frac{2 c_{3}}{v^{2}}}}=\frac{\frac{-2 u^{3}}{v^{4}}}{v^{4}} \cdot \frac{-v^{3}}{2 c_{3}}=\frac{-u^{3}}{c_{3} v^{2}} \\
& \Gamma_{22}^{2}=\frac{\mathrm{EG}_{\mathrm{v}}-2 \mathrm{FF}_{\mathrm{v}}+\mathrm{FG}_{\mathrm{u}}}{2\left(\mathrm{EG}-\mathrm{F}^{2}\right)}= \\
& \frac{\frac{\mathrm{c}_{1}}{\mathrm{u}^{2},} \frac{2 \mathrm{u}^{2}}{\mathrm{v}^{3}} \frac{2 \mathrm{c}_{2}}{\mathrm{v}}, \frac{-\mathrm{c}_{2}}{\mathrm{v}^{2}} \frac{\mathrm{c}_{2}}{\mathrm{v}}, \frac{2 \mathrm{u}}{\mathrm{v}^{2}}}{\frac{2 \mathrm{cf}}{\mathrm{v}^{2}}}=\frac{\frac{-2 \mathrm{c}_{1} \mathrm{u}^{2}}{\mathrm{u}^{2} \mathrm{v}^{3}}\left|\frac{2 \mathrm{c}_{2}^{2}}{\mathrm{v}^{3}}\right| \frac{2 \mathrm{uc}_{2}}{\mathrm{v}^{3}}}{\frac{2 \mathrm{c}_{\mathrm{g}}}{\mathrm{v}^{2}}}= \\
& \frac{\frac{-2 c_{1} u^{2}+2 u^{2} c_{2}^{2}+2 u^{3} c_{2}}{u^{2} v^{3}}}{\frac{2 c_{3}}{v^{2}}}=\frac{2 c_{1} u^{2}\left|2 u^{2} 2 c_{2}^{2}\right| 2 u^{3} c_{2}}{u^{2} v^{3}} \cdot \frac{v^{2}}{2 c_{3}}= \\
& \frac{\left(\mathrm{c}_{1}\left|\mathrm{c}_{2}^{2}\right| \mathrm{uc}_{2}\right)}{\mathrm{vc}_{3}}=\frac{\mathrm{uc}_{2} \mathrm{c}_{3}}{\mathrm{C}_{3} \mathrm{v}}
\end{aligned}
$$

We take the left hand side of equation (C):

$$
\begin{aligned}
& \mathrm{K}=\frac{1}{\mathrm{D}}\left[\frac{\partial}{\partial \mathrm{v}}\left(\frac{\mathrm{D}}{\mathrm{E}} \Gamma_{11}^{2}\right)-\frac{\partial}{\partial \mathrm{u}}\left(\frac{\mathrm{D}}{\mathrm{E}} \Gamma_{12}^{2}\right)\right]= \\
& \frac{1}{\sqrt{\sqrt{\mathrm{EG}-\mathrm{F}^{2}}}}\left[-\frac{\partial}{\partial \mathrm{u}}\left(\frac{\sqrt{\mathrm{EG}-\mathrm{F}^{2}}}{\mathrm{E}} \Gamma_{12}^{2}\right)\right]= \\
& \frac{\sqrt{\mathrm{v}}}{\sqrt{\mathrm{C}_{3}}}\left[-\frac{\partial}{\partial \mathrm{u}}\left(\frac{\sqrt{\mathrm{C}_{\mathrm{g}}}}{\mathrm{v}}, \frac{\mathrm{u}^{2}}{\mathrm{C}_{1}}, \frac{\mathrm{c}_{1}}{\mathrm{C}_{3} \mathrm{u}}\right)\right]=\frac{\mathrm{v}}{\sqrt{\mathrm{C}_{3}}}\left[-\frac{\partial}{\partial \mathrm{u}}\left(\frac{\mathrm{u}}{\mathrm{v} \sqrt{\mathrm{C}_{3}}}\right)\right]= \\
& \frac{\mathrm{v}}{\sqrt{\mathrm{C}_{3}}} \cdot \frac{-1}{\mathrm{v} \sqrt{\mathrm{C}_{3}}}=\frac{-1}{\mathrm{C}_{3}}=-\frac{1}{1.644934067}=-0.607927101
\end{aligned}
$$

Now, we take the right hand side of equation ( C):

$$
\begin{aligned}
& \mathrm{K}=\frac{1}{\mathrm{D}}\left[\frac{\partial}{\partial \mathrm{u}}\left(\frac{\mathrm{D}}{\mathrm{G}} \Gamma_{22}^{1}\right)-\frac{\partial}{\partial \mathrm{v}}\left(\frac{\mathrm{D}}{\mathrm{G}} \Gamma_{12}^{1}\right)\right]= \\
& \frac{1}{\sqrt{\mathrm{EG}-\mathrm{F}^{2}}}\left[\frac{\partial}{\partial \mathrm{u}}\left(\frac{\sqrt{\mathrm{EG}-\mathrm{F}^{2}}}{\mathrm{G}} \Gamma_{22}^{1}\right)\right]= \\
& \frac{1}{\sqrt{\mathrm{C}_{3}}}\left[\frac{\partial}{\partial \mathrm{u}}\left(\frac{-\mathrm{u}}{\mathrm{v} \sqrt{\mathrm{C}_{3}}}\right)\right]=\frac{\mathrm{v}}{\sqrt{\mathrm{C}_{3}}} \cdot \frac{-1}{\mathrm{v} \sqrt{\mathrm{C}_{3}}}=\frac{-1}{\mathrm{C}_{3}}= \\
& -\frac{1}{1.644934067}=-0.607627101
\end{aligned}
$$

This result shows that Weibull distribution converges to the normal distribution.

\section{CONCLUSION}

By using equation (C), we find the Gaussian curvature for Weibull distribution equal to that means this distribution converges to normal distribution.

\section{ACKNOWLEDGEMENT}

Praise is to Allah for helping me to complete this research. My thanks are due to my family for their spiritual support during the period of writing this research.

\section{REFERENCES}

Barndorff-Nielsen, O.E., D.R. Cox and N. Reid, 1986. The role of differential geometry in statistical theory. Int. Stat. Rev., 54: 83-96.

Chen, W.W.S., 2014. Finding gaussian curvature of lifespan distribution. Applied Math., 5: 3392-3400.

Do Carmo, M.P., 1976. Differential Geometry of Curves and Surfaces. Prentice Hall, New Jersey, USA., ISBN:9780132125895, Pages: 503.

Kass, R.E. and P.W. Vos, 1997. Geometrical Foundations of Asymptotic Inference. Wiley, Hoboken, New Jersey, USA., ISBN: 9780471826682, Pages: 355.

O' Neill, B., 1966. Elementary Differential Geometry. Academic Press, Inc., Orland, Florida, USA., Pages: 411.

Wikipedia, 2005. Fisher information metric. Wikimedia Foundation Inc., San Francisco, California, USA. https://en.wikipedia.org/wiki/Fisher_information_met ric. 